Article

\title{
Elastic Full-Waveform Inversion Using Migration-Based Depth Reflector Representation in The Data Domain
}

\author{
Vladimir Tcheverda ${ }^{1,2, *}$ and Kirill Gadylshin ${ }^{1,2}$ \\ 1 Institute of Petroleum Geology and Geophysics SB RAS, Novosibirsk, Russia; \\ 2 Institute of Computational Mathematics and Mathematical Geophysics, SB RAS, Novosibirsk, Russia \\ * Correspondence: CheverdaVA@ipgg.sbras.ru; Tel.: +7-913-474-9979
}

\begin{abstract}
Full-waveform seismic data inversion has given rise to hope for the simultaneous and automated execution of tomography and imaging by solving a nonlinear least-squares optimization problem. As previously recognized, brute force minimization by classical methods is hopeless if the data lacks low temporal frequencies. The article developed a reliable numerical method for recovering smooth velocity using model space decomposition. We present realistic synthetic examples to test the presented algorithm.
\end{abstract}

Keywords: FWI, macro velocity, waveform inversion, optimization, reflected waves

\section{Introduction}

The velocity model building in the depth domain is necessary to ensure correct travel times and is, therefore, an essential element of modern seismic data processing. Back in the mid-80s of the last century, A. Tarantola presented full-waveform inversion (FWI), based on a comparison of observed and synthetic seismograms. The L2 norm is widely used for this comparison, although other criteria are being considered. For the minimization of the objective function and finding the elastic parameters of the subsurface, iterative gradient algorithms are usually applied. This approach to FWI, initially proposed by Lailly [1] and Tarantola [2], has been developed and studied in a large number of publications (see [3] and references therein). However, straightforward application of FWI reliably restores only the reflectivity component of the subsurface, but cannot provide a smooth velocity model. The smooth component could not be stably restored without the presence of extremely low temporal frequencies. The fact is that the form of the data mismatch functional differs greatly depending on the various velocity components - it is almost quadratic concerning the reflectors, but perturbations of the smooth velocity component (propagator) lead to very complex and nonlinear behaviour (see, For example, [4] ). Heuristically, this is due to the so-called "cycle-skip" problem, where phase shifts between recorded and synthetic data produce local minima. In paper [5], the authors introduce new inversion strategy based on the use of increasing temporal frequencies: they start the inversion at the lowest available frequencies, and at each subsequent iteration, they increase the frequency and use the results of the previous step as an initial guess. But they perform the time-domain inversion, so they need to use the low-pass time-domain band-pass filtering at each stage, which reduces the effectiveness of this method. The next step in the implementation of FWI in the frequency domain with increasing time frequencies was made by G. Pratt et al. in [6]. They use minimization in the time-frequency domain and move very naturally from low to high frequencies. The key to the successful implementation of this approach is an 
efficient solver for the stationary Helmholtz/elastic wave equation. Unfortunately, this approach did not solve the problem of stable macro-velocity recovery with a lack of low frequencies in the data for both surface and borehole surveys (vertical seismic profile) ([4], [7]). Of course, on this basis, they enhance the role of the existing low temporal frequencies and, in a sense, improve the stability of the propagator reconstruction, but they cannot offer a stable solution to the problem. The alternative to this standard formulation was proposed in the paper [8]. The main message of this alternative is the modification of the data misfit functional based on the decomposition of a model space into two subspaces:

- the subspace of smooth propagators (macro velocity components);

- the subspace of spatial reflectors, which, in turn, is the image of the subsurface.

This approach was modified for the frequency domain in [9] and was applied for reconstruction of well-known Marmousi model for realistic frequency bandwidth and offsets. In this paper, the implementation of this approach for elastic Full Waveform Inversion is presented.

2. Method and theory

\subsection{Data Space Reflectivity FWI reformulation}

The seismic inverse problem is equivalent to the solution of the nonlinear operator equation:

$$
F(m)=d,
$$

where $F: M \rightarrow D$ is a nonlinear forward operator, associated with the elastic waves equation, which maps model space $M$ into data space $D$.

As in acoustic MBTT case [8] the essence of this formulation is to decompose the velocity model into two constituents: smooth propagators $p$ and oscillating reflector $r$. Since we search for both $V_{p}$ and $V_{s}$ velocity models, in fact, $p$ is a vector $p=\left(p V_{p}, p V_{s}\right)$, where $p V_{p}$ - P-velocity propagator and $p V_{s}$ - S-velocity propagator. In turn, the reflector is treated as the result of the true amplitude migration applied to the part of the data called data-space reflectivity (DSR) - preimage of the depth reflector in data space:

$$
m=p+r=M(p) s .
$$

The critical moment of this decomposition is propagator-reflector interrelation $r=M(p) s$ with operator $M(p)$ being some kind of true-amplitude prestack migration/linearized inversion. In particular, reweighted version of adjoint operator based migration:

$$
M(p) s=W \circ \operatorname{Re}\left\{D F^{*} s\right\}
$$

Here $D F$ is Frechet derivative of the full nonlinear map $F,^{*}$ means the adjoint operator and $W$ is some linear operator providing true amplitudes imaging/migration. This kind of model space decomposition leads to the following modified data misfit functional:

$$
E(p, s)=\|F(p+M(p) s)-d\|_{D}^{2} .
$$

Minimization with respect to a propagator $\mathrm{p}$ and a data-space reflectivity $\mathrm{s}$ is performed independently and by turn. We start with admitting $s=d$ and search for some intermediate value of propagator $p$. After stabilization of this process, the search is switched to data-space reflectivity $s$ and so on.

We implemented the search for the propagator using Gauss-Newton method [6]. Since the dimension of propagator space is small enough concerning the size of the whole model space $M$, we can calculate partial SVD (only highest singular vectors which correspond the highest singular 
values) of the approximate Hessian $\mathcal{H}_{a}$ on the first iteration using matrix-free methods (such as Krylov-subspace based methods, for numerical implementation see [10]). On the next step, we use r-pseudoinverse of this operator [11] as a preconditioner in the Conjugate-Gradient method during propagator minimization:

$$
\begin{gathered}
p_{k+1}=p_{k}+\mu_{k} S_{k}, \quad S_{0}=\nabla_{0}, \\
S_{k}=-P \nabla_{k}-\frac{\left\langle P \nabla_{k}, \nabla_{k}-\nabla_{k-1}\right\rangle_{M}}{\left\langle P \nabla_{k-1}, \nabla_{k-1}\right\rangle_{M}} S_{k-1},
\end{gathered}
$$

where $p_{k}=\left(p V_{p}, p V_{s}\right)$ - propagator on k-th iteration, $P=\left(\mathcal{H}_{a}\right)_{r}^{\dagger}-$ pseudoinverse of the approximate Hessian. The gradient $\nabla_{k}$ with respect to the propagator unknown is calculated as follows:

$$
\nabla_{k}=\operatorname{Re}\left\{D F^{*} \delta d_{k}+\left(D^{2} F\left(p_{k}\right) W^{*} D F^{*} \delta d_{k}\right)^{*} s\right\},
$$

here $\delta d_{k}=F\left(m_{k}\right)-d$ is data residual on current iteration, $D F$ - a first derivative of the forward map calculated at the point $m_{k}=p_{k}+M\left(p_{k}\right) s$ and $D^{2} F$ - second derivative calculated at the point $p_{k}$. Incorporation into inversion process Hessian $\mathcal{H}_{a}$ helps to mitigate crosstalk between $V_{p}$ and $V_{s}$ models. Minimization of the data-space reflectivity $s$ is done via steepest descent method. The corresponding gradient $\nabla_{s}$ is calculated as follows:

$$
\nabla_{s}=M^{*}(p) D F^{*} \delta d,
$$

where $M^{*}(p)$ - adjoint to the migration operator, also known as the demigration.
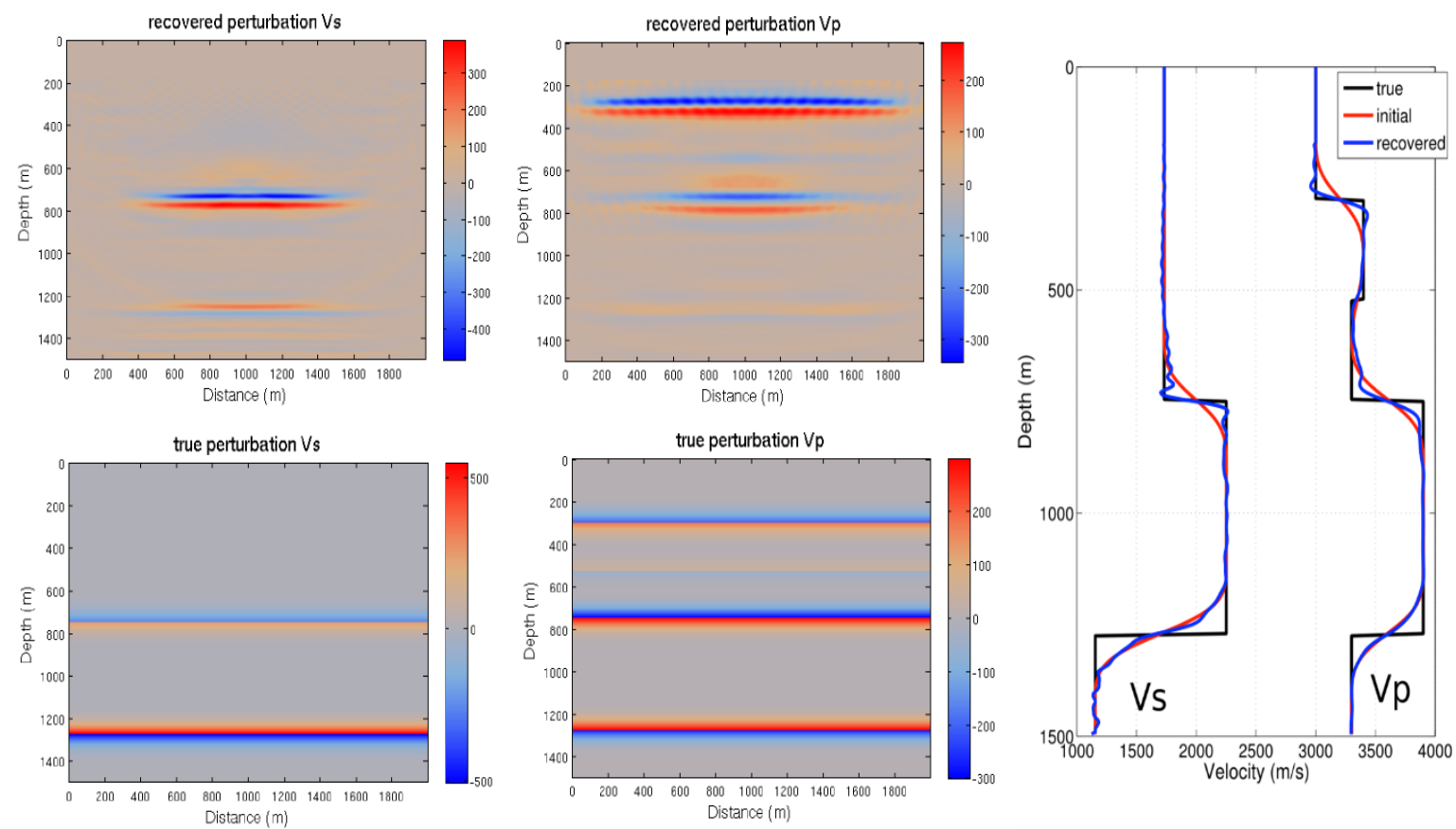

Figure 1.: Minimization with respect to data-space reflectivity: comparison of recovered depth reflectors (top) with the true image (bottom) for Vs (left) and Vp (right)

Figure 2.: Vertical profiles of the true velocity distribution (black) at a distance $1000 \mathrm{~m}$, smooth propagator used for inversion (red) and the reconstructed full velocity model after minimization concerning data-space reflectivity (blue) 


\subsection{The validation of propagator/reflector decomposition}

The first question to be answered is the possibility of representing the depth reflector $r=$ $\left(r V_{p}, r V_{s}\right)$ through the migration operator with current propagator $p=\left(p V_{p}, p V_{s}\right)$. To assess the feasibility of this representation, we consider the vertically inhomogeneous layered medium (see Fig. 1). To evaluate the crosstalks between $V_{p}$ and $V_{s}$ models during minimization of modified misfit function $E(p, s)$ with respect to data-space reflectivity the targets horizons for $V_{p}$ and $V_{s}$ we choose models such that they don't match in the upper part. Input data are synthesized for the set of ten uniformly sampled frequencies in the range 5-30 Hz. The acquisition system has 25 vertical force point sources and 100 2C-receivers located at depth $10 \mathrm{~m}$ with a lateral spacing of $80 \mathrm{~m}$ and $20 \mathrm{~m}$ respectively. For the sake of simplicity in the definition of migration operator, we use the identity weight matrix $\mathrm{W}=\mathrm{Id}$, that is, we do not use true-amplitude reweighting in this experiment. Propagators $p V_{p}$ and $p V_{s}$ (see Fig.1, red plots) provide the information on macro velocity - we are concentrated only on the reconstruction of space reflectivity.

During minimization process, we performed 30 nonlinear CG iterations with respect to data-space reflectivity unknown. The results of inversion are presented on Figs. 1 and 2. As one can clearly observe there are no crosstalks effects between $V_{p}$ and $V_{s}$ models and the depth reflectors are sitting in the correct positions.

\subsection{Choice of depth reflectivity parametrization}

The second question is related to the parametrization of the depth reflectivity. Which elastic model parametrization is better for spatial reflectivity in the context of the inverse problem? Consider two different parameterizations for the depth reflectivity: $r_{1}=\left(r V_{p}, r V_{s}\right)$ and $r_{2}=$ $\left(r I_{p}, r I_{s}\right)$, where $I_{p}=\rho V_{p}, I_{s}=\rho V_{s}$ - elastic impedances, $\rho$ is a density. For a fixed acquisition geometry and reference model $m_{0}$ one may consider two linearized forward maps $L_{1}=\frac{\partial F}{\partial m_{1}}$ and $L_{2}=\frac{\partial F}{\partial m_{2}}$ - first Frechet derivatives of the forward map $F$ concerning the different elastic model parametrizations, evaluated at the point $m_{0}$. Following [12] the comparative resolution analysis of singular value decomposition (SVD) of two linear maps $L_{1}$ and $L_{2}$ will answer the question. The numerically calculated singular spectrums are presented on Fig. 3. As it can be clearly observed, the



Figure 3.: Singular spectrums of linearized forward maps for parametrization through velocities (red) and elastic impedances (blue) 
linearized inverse problem for parametrization $\left(\boldsymbol{V}_{\boldsymbol{p}}, \boldsymbol{V}_{\boldsymbol{s}}\right)$ is better conditioned (see Fig. 3) than in case of elastic impedances. Next step is resolution analysis. Following [12] for a fixed condition number of truncated operator, we construct the projection on stable subspaces for both operators. We built projections for a fixed condition number of truncated operator equal to $\mathbf{1 0}^{\mathbf{1}}$ (see Figures $4,5,6$ ). As one may recognize, there is no significant difference in projections for both parametrization, i.e. they are all can be used for depth reflector reconstruction. We find it more natural to choose $\left(\boldsymbol{V}_{\boldsymbol{p}}, \boldsymbol{V}_{\boldsymbol{s}}\right)$ as elastic model parametrization.

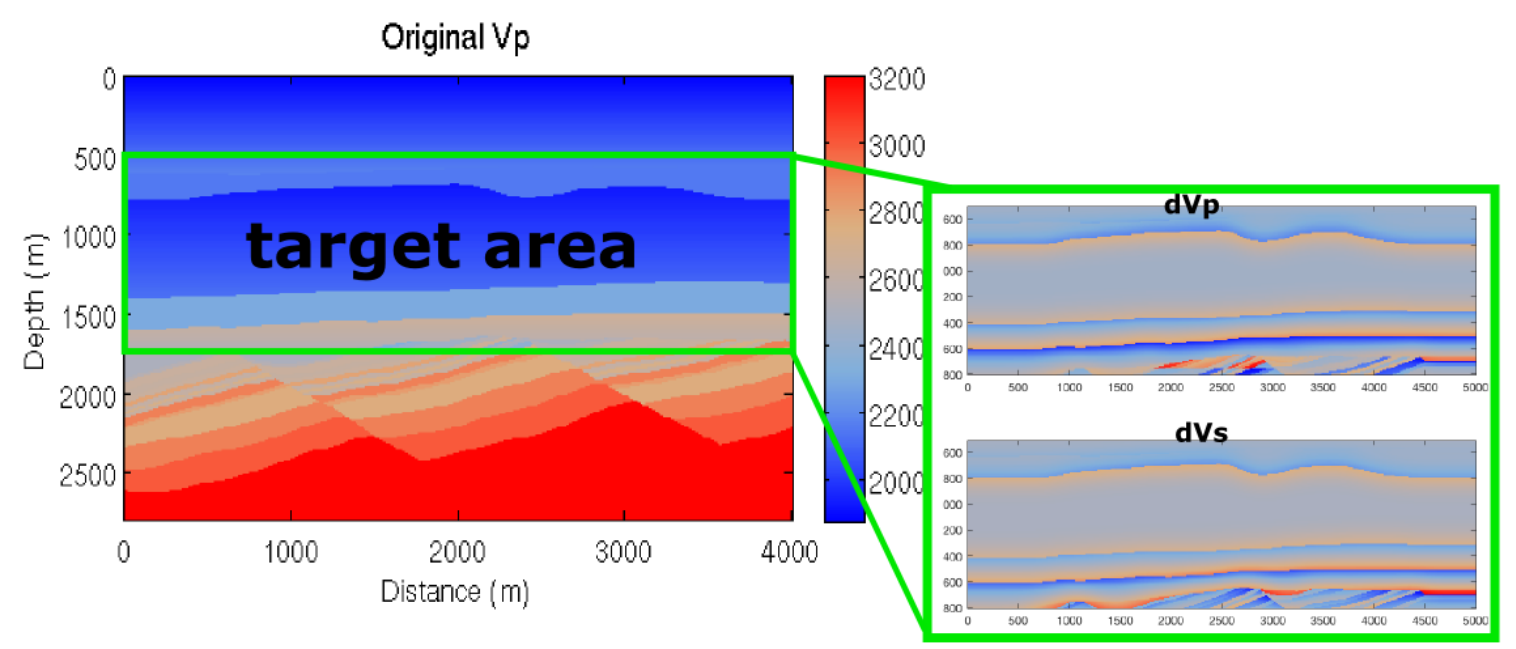

Figure 4.: Reference $V_{p}$ model (left) and true depth reflectivity perturbations $\left(r V_{p}, r V_{s}\right)$ on the right
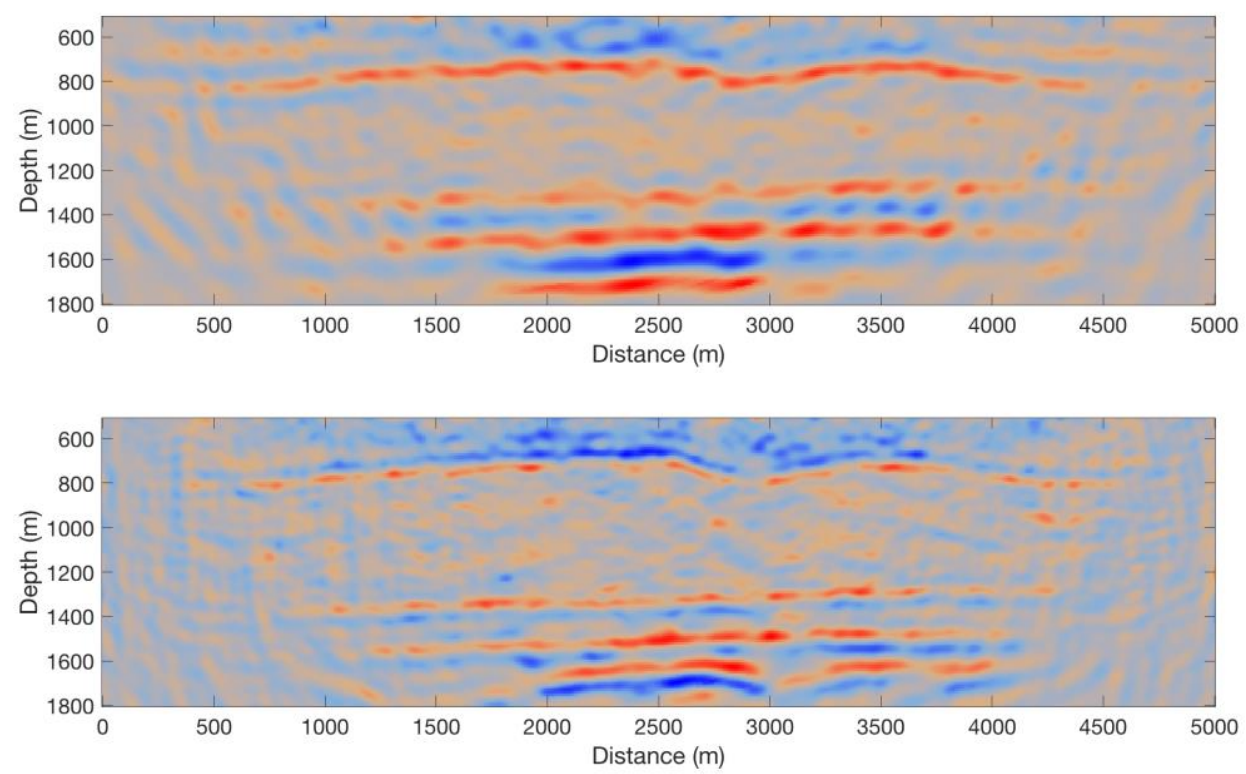

Figure 5.: Stable components (projection onto the highest right singular vectors) of depth reflector reconstruction for $\left(V_{p}, V_{s}\right)$ parametrization. Top $-r V_{p}$ projection, bottom $-r V_{s}$ the projection for the truncated operator condition number $10^{1}$ 

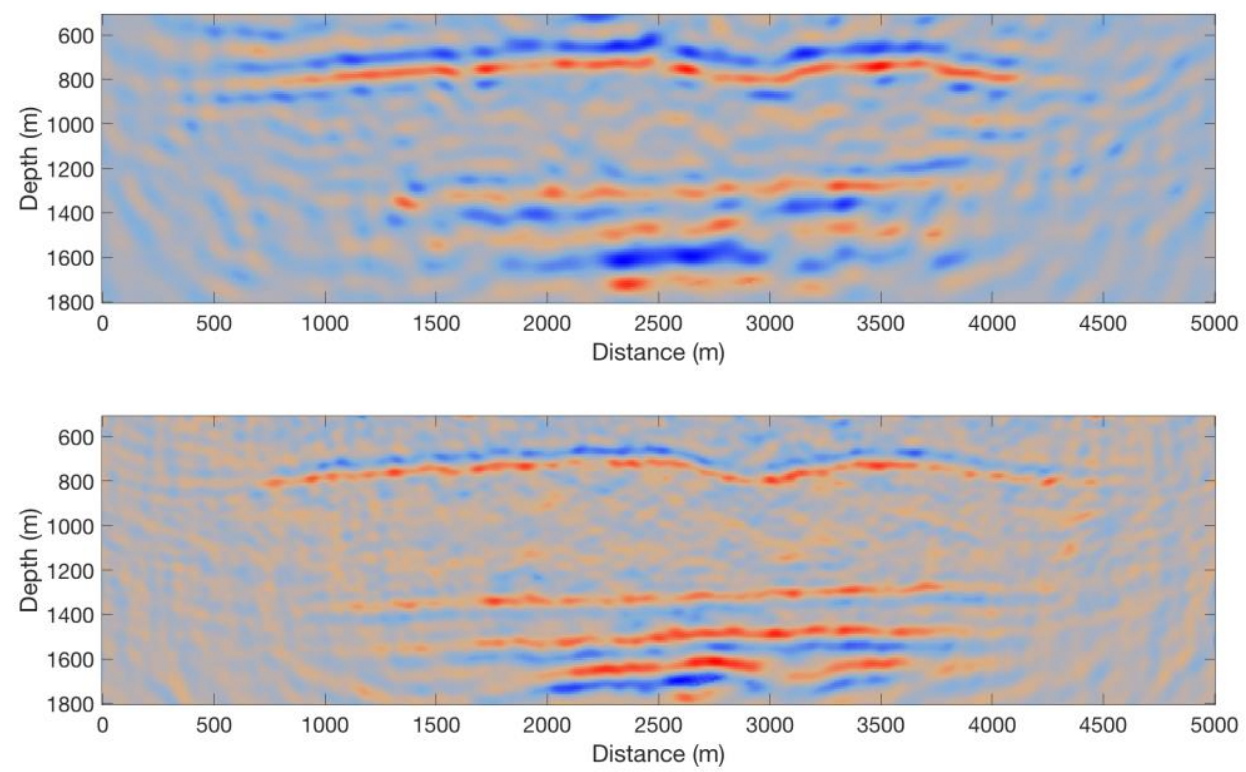

Figure 6.: Stable components (projection onto the highest right singular vectors) of depth reflector reconstruction for $\left(I_{p}, I_{s}\right)$ parametrization. Top $-r I_{p}$ projection, bottom $-r I_{s}$ the projection for the truncated operator condition number $10^{1}$

\section{Results}

\subsection{Experiment 1}

The realistic example invoked in the study is based on the part of SEG/EAGE overthrust synthetic $V_{p}$-velocity model, the $V_{s}$ is a scaled version of $V_{p}$ model (by a factor $1 / \sqrt{3}$ ). Input data are synthesized for the set of ten uniformly sampled frequencies in the range $5 \div 10 \mathrm{~Hz}$. The acquisition system has 31 vertical point force sources and $4002 \mathrm{C}$-receivers located at surface $\mathrm{z}=0 \mathrm{~m}$ with a lateral spacing of $325 \mathrm{~m}$ and $25 \mathrm{~m}$ respectively. Initial guess for the propagator is a vertically heterogeneous model (see Fig. 7). As an initial guess for the data-space reflectivity variable $s$ we used the observed data itself.

The results of elastic Full Waveform Inversion in the modified formulation are presented in Fig. 7. The search for a propagator is done in the space of 2D B-Splines functions of order 3. In this way one may guarantee that the search is implemented in the smooth propagator space. We perform the minimization using the projected conjugate gradient method, where orthogonal projector onto smooth space (B-splines) is used as a projection onto the feasible set.

\subsection{Experiment 2}

In the next experiment, we use the Gullfaks South [13] model to validate the approach based on propagator/reflector decomposition. 2D elastic model one can see in Figure 8 (left column). We would like to pay the attention that $\gamma=\frac{V_{p}}{V_{s}}$ is not a constant, that is there no dependence between $V_{p}$ and $V_{S}$. Input data are generated for the set of 19 uniformly sampled frequencies in the range $5-$ $20 \mathrm{~Hz}$. The acquisition system has 39 vertical point force sources and $2002 \mathrm{C}$-receivers located at surface $z=0 \mathrm{~m}$ with a lateral spacing of $100 \mathrm{~m}$ and $20 \mathrm{~m}$ respectively. We start with the depth-dependent initial model (Fig 8., middle column), which is far from the real velocities. 2D B-Splines of order 3 was used as the basis of the smooth propagator space. Doing minimization, 
finally, we come to the result which fairly well coincides with the original model for all three parameters: $V_{p}, V_{s}$ and $\gamma$ (Fig. 8, right column).
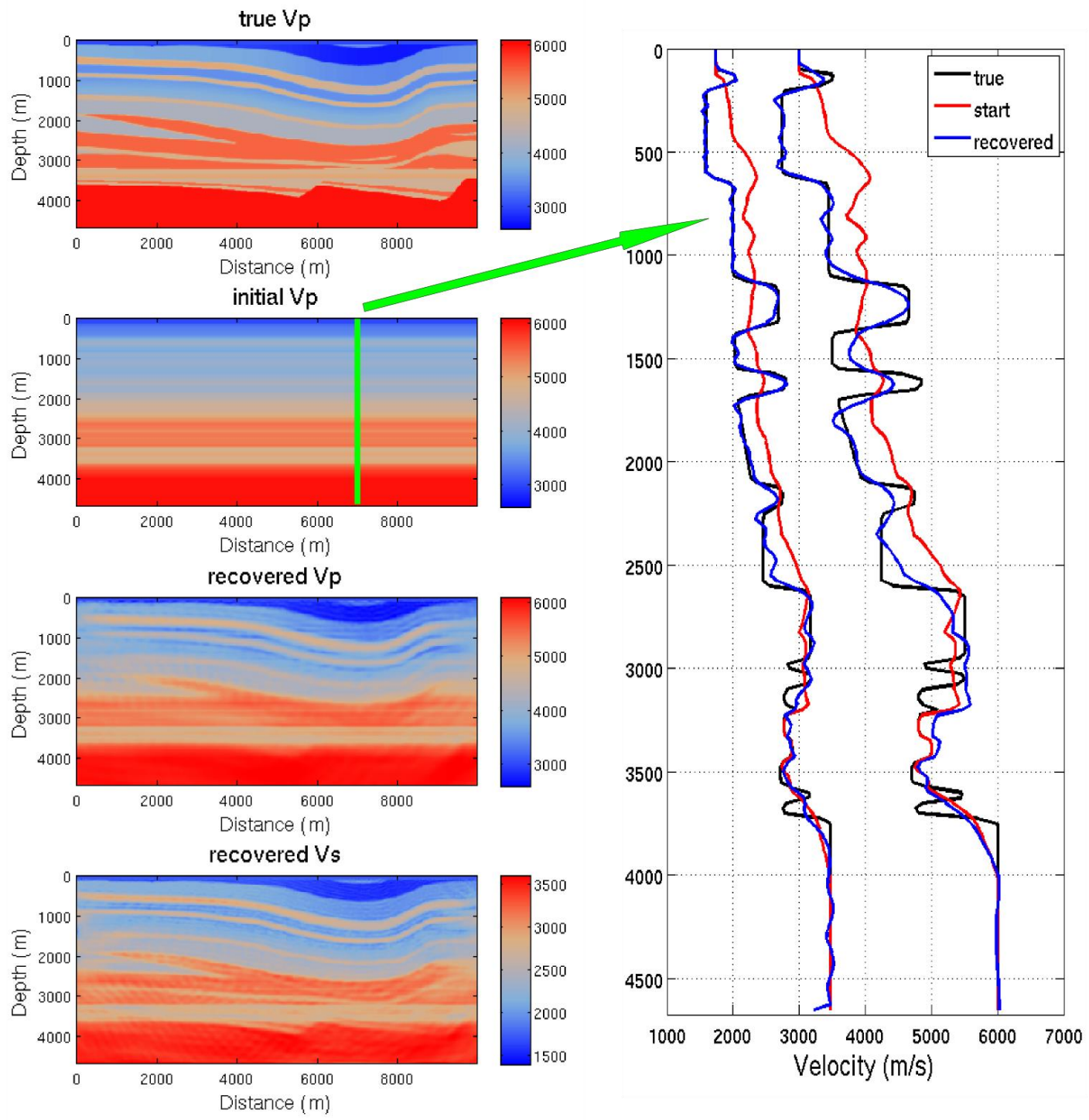

Figure 7.: Elastic FWI in the modified formulation results. On the left (from top to bottom): true Vp model, initial Vp model, inverted Vp and inverted Vs distributions. On the right: vertical profiles at the distance 7000m for Vp- and Vs-velocity distributions (black plot - true velocity, red - initial and blue - inverted velocities)

The data comparison is demonstrated on Fg. 9. Synthetics are generated for the shot located at $x=$ $700 \mathrm{~m}$, and the source wavelet is a Ricker with dominant frequency $10 \mathrm{~Hz}$. As it can be clearly observed, the reconstructed velocity model describes main part of the data for both vertical and horizontal receiver components.

\subsection{Experiment 3}

Finally, we consider a realistic synthetic example based on the Marmousi2 elastic velocity model [14]. Input data are synthesized in the frequency domain for the set of twenty uniformly sampled frequencies in the range $5-15 \mathrm{~Hz}$. We choose the lowest temporal frequency equal to $5 \mathrm{~Hz}$ following some publications and discussions with colleagues dealing with real seismic data. The acquisition system has 46 volumetric sources and 460 receivers located at the top of the model with lateral spacing of $200 \mathrm{~m}$ and $20 \mathrm{~m}$ respectively. As the initial guess for propagator, we use the 
vertically heterogeneous model (see Fig. 10). As an initial guess for the time reflectivity variable $s$ is used the observed data itself.
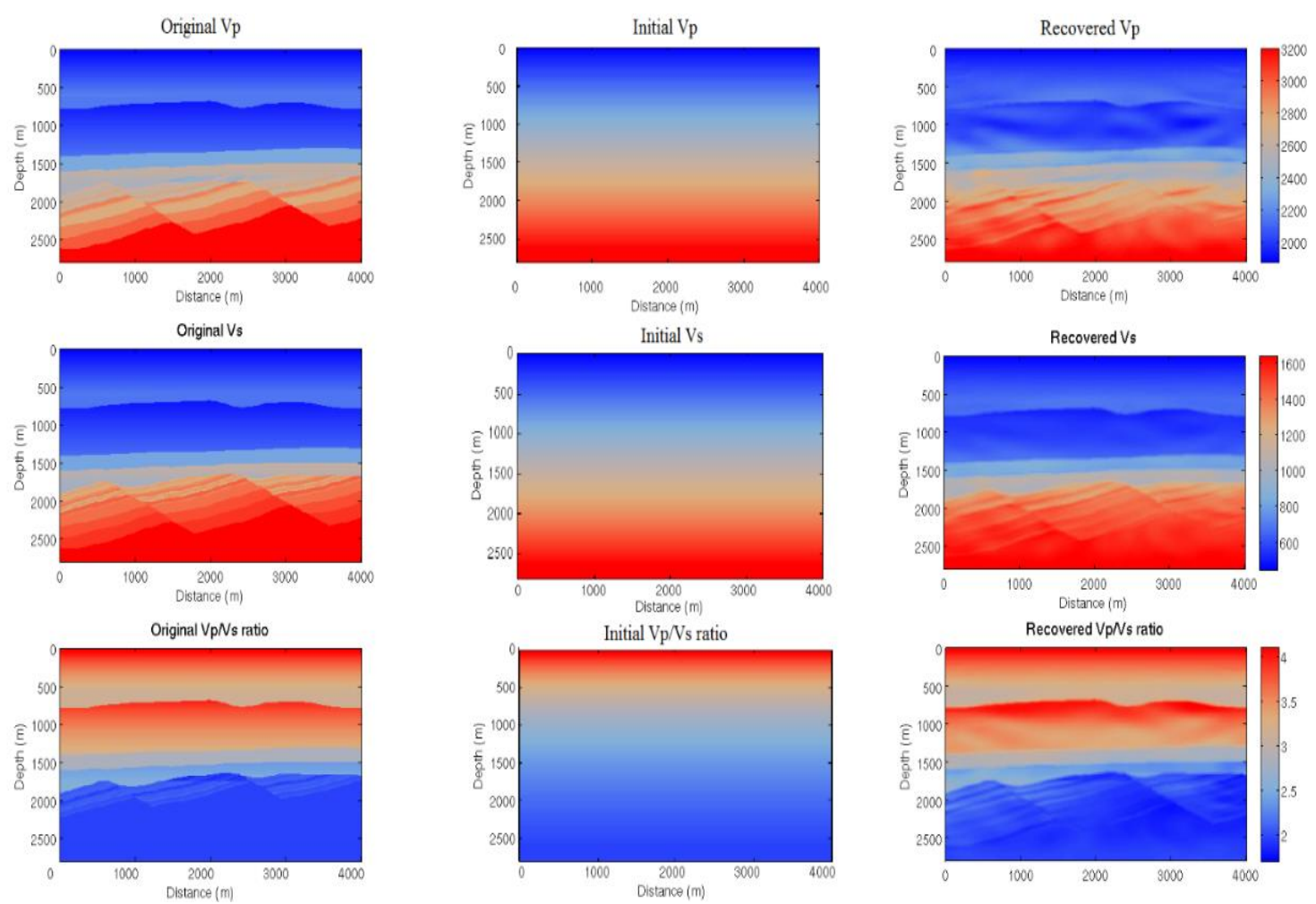

Figure 8. : Gullfaks velocity model. Left column from top to bottom: original Vp, Vs and Vp/Vs models. Middle column: initial Vp, Vs and Vp/Vs models. Right column: reconstructed Vp, Vs and $\mathrm{Vp} /$ Vs velocity models after DSR FWI.
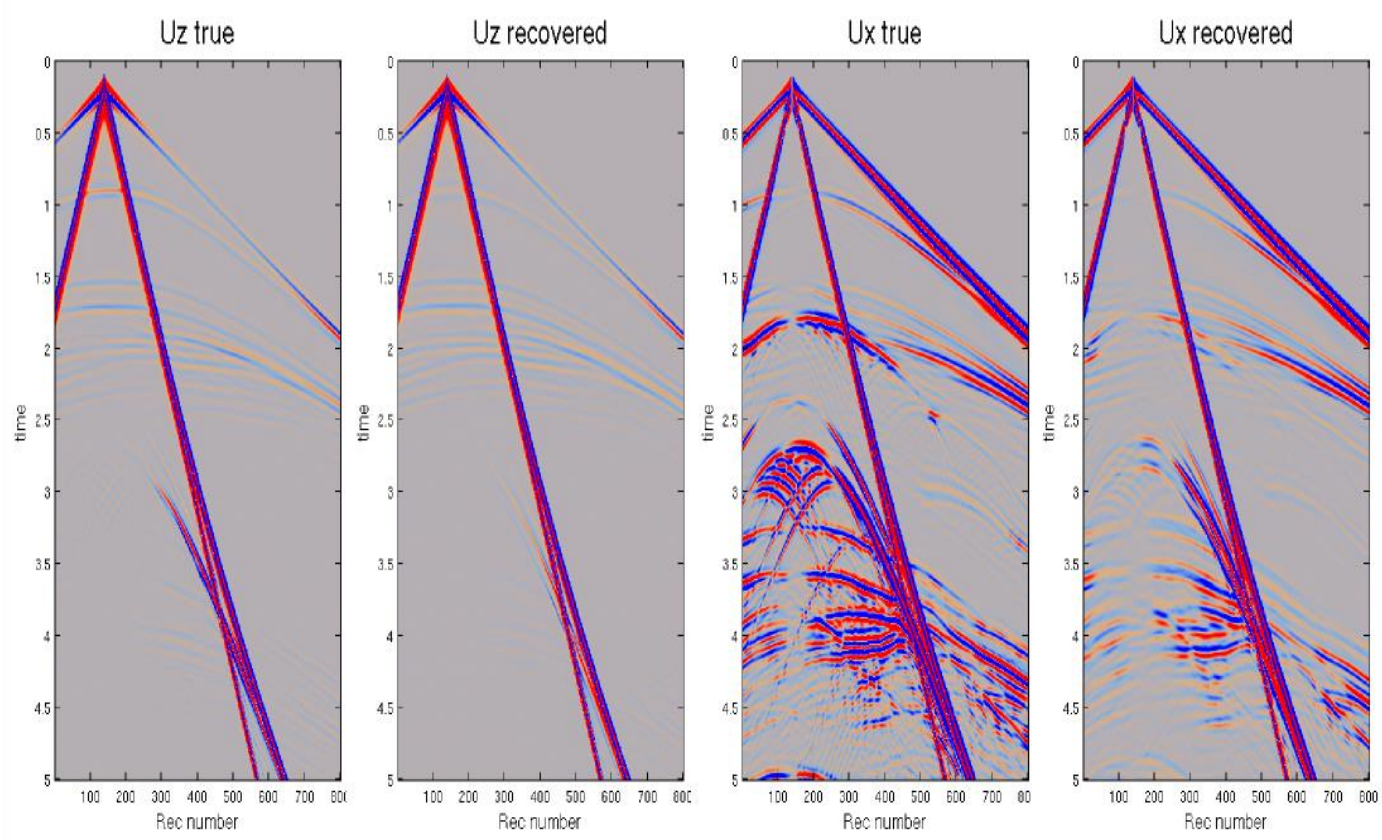

Figure 9. : Synthetics generated for shot located at position $x=700 \mathrm{~m}$. Source wavelet - Ricker with dominant frequency $10 \mathrm{~Hz}$. From left to right: vertical component for the true model and for the recovered model, horizontal component for the true model and recovered model 

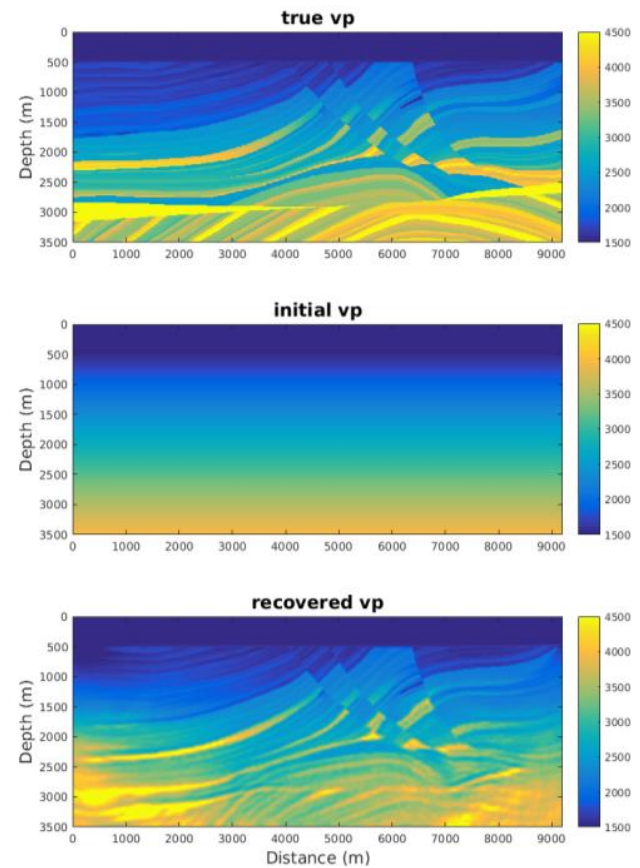

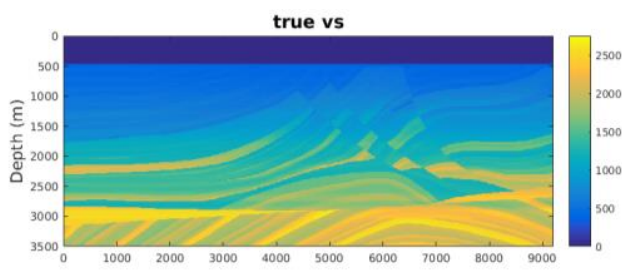

initial vs
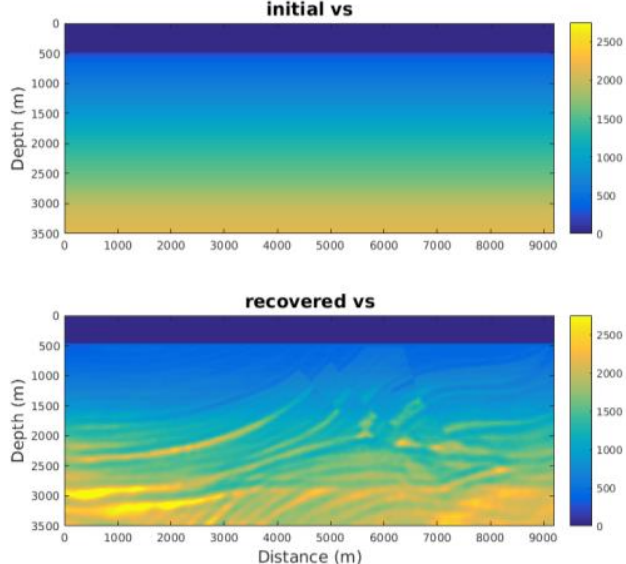

Figure 10. : a),b) exact Marmousi2 $V_{p}$ and $V_{s}$ models; c),d) initial guess for $V_{p}$, and $V_{s}$; e),f) reconstructed $V_{p}$ and $V_{s}$ models through DSR FWI algorithm

We present the results of DSR FWI in Fig. 10. As in the previous example, we search for the propagator in the space of 2D B-Splines functions of order 3 . This guarantees the smoothness of the solution and reasonable size of the model space. To minimize the cost function, we use the projected conjugate gradient method, with orthogonal $\mathrm{L}_{2}$-projector onto smooth space (B-splines). To compute true-amplitude migration $\mathrm{M}$, we apply the imaging procedure on the base of inverse generalized Radon transform with the representation of the Green's function as decomposition of Gaussian beams. As one can see, the local reconstruction of the Marmousi velocity model is good enough. Next, to evaluate the quality of the macro velocity (propagator) reconstruction, we generate common image gathers (CIGs) in the offset-depth domain (see Fig. 11). These CIGs are computed for the initial $V_{p}$ model and for the model reconstructed via DSR FWI. The reconstructed model significantly improves the flatness of the events in the CIGs concerning the initial model CIGs.

\section{Conclusions}

DSR FWI modified data misfit functional on the base of propagator/reflector decomposition of the model space were introduced and investigated. The comparative SVD resolution analysis of different elastic model parameterizations demonstrates no principal differences between velocities and elastic impedances as unknowns in the context of the inverse problem. This decomposition provides an alternative way to perform the full-waveform inversion. Inversion is performed separately for the search of smooth macro velocity (reduced model dimension) and sharp reflectors (classical true amplitude prestack migration for known macro velocity). By this approach, we succeeded to reconstruct the Gullfaks South model and the Marmousi2 model without low time frequencies - we used frequencies from $5 \mathrm{~Hz}$ to $20 \mathrm{~Hz}$ only and do not use huge offsets. 


\section{ClGs initial}

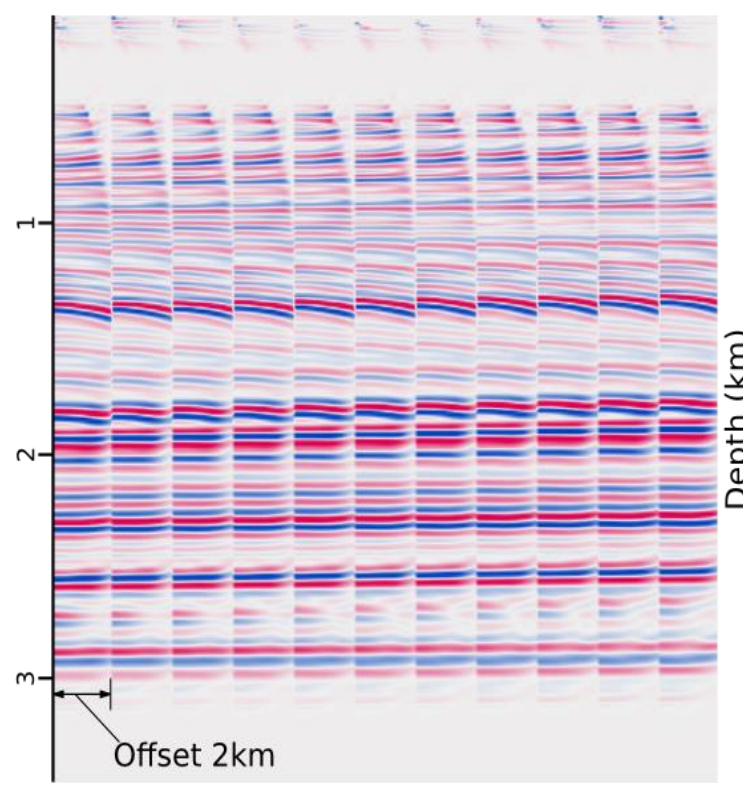

CIGs after DSR FWI

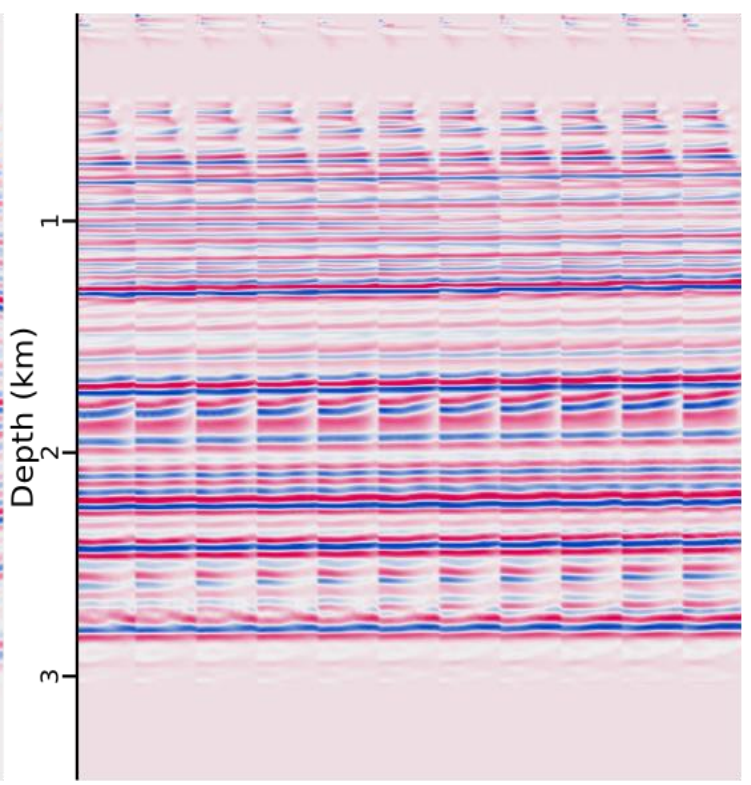

Figure 11.: Common image gathers (CIGs) at positions $X=[3.5: 0.01: 3.6] \mathrm{km}$ from the initial guess (left) and model obtained via DSR FWI

Funding: This research was funded by Russian Science Foundation project number 20-11-20112.

Acknowledgments: The authors are grateful to Professor Guy Chavent for inspiring discussions on full-waveform inversion, making valuable comments and supporting during the research study.

Conflicts of Interest: The authors declare no conflict of interest

\section{References}

1. Lailly, P.; The seismic inverse problem as a sequence of before stack migrations. Conference on Inverse Scattering: Theory and Application. SIAM. 206 - 220. 1983.

2. Tarantola, A.; Inversion of seismic reflection data in the acoustic approximation. Geophysics 1984.

3. Virieux, J.; Operto, S.; An overview of full-waveform inversion in exploration geophysics. 2009.

4. Sirgue, L.; The importance of low frequencies and large offset in waveform inversion. 68th EAGE Technical conference and Exhibition, A037. 2006.

5. Bunks, C.; Saleck, F.M.; Zaleski, S.; Chavent, G. Multiscale seismic inversion. Geophysics, 1995, V. 60, iss.5. P. 1457-1473.

6. Pratt, G.; Shin, C.; Hicks, G.J. . Gauss-Newton and full Newton methods in frequency-space seismic waveform inversion Geophysical Journal International. 1998, V. 133, iss. 2. P. 341-362.

7. Silvestrov, I.; Neklyudov, D.; Kostov, C.; Tcheverda, V.; Full-Waveform Inversion for Macro Velocity Model Reconstruction in Look-Ahead Offset Vertical Seismic Prfile: Numerical Singular Value Decomposition-Based Analysis. Geophysical Prospecting, 61(6): 1099 - 1113. 2013.

8. Clement F.; Chavent G.; Gomez, S. Migration-based traveltime waveform inversion of 2-D simple structures: A synthetic example Geophysics. 2001, V. 66. P. 845-860.

9. Tcheverda, V.; Chavent, G.; Gadylshin, K. Macrovelocity reconstruction by reflection FWI, 78th EAGE Conference \& Exhibition, Vienna, Austria, 30 May-2 June 2016. Th SRS2 06.

10. Hernandez, V.; Roman, J. E.; Vidal, V. SLEPc: A scalable and flexible toolkit for the solution of eigenvalue problems ACM Trans. Math. Software. 2005, V. 31, iss. 3. P. 351-362.

11. Cheverda, V.A.; Kostin, V.I. R-pseudoinverses for compact operators in hilbert spaces: existence and stability Journal of Inverse and Ill-posed Problems. 1995, V. 3, iss. 2. P. 131-148.

12. Gadylshin, K.; Bakulin, A.; Dmitriev, M.; Golikov, P.; Neklyudov, D.; Tcheverda, V.; Effect of free surface related multiples on near surface velocity reconstruction with acoustic frequency domain FWI 76th 
European Association of Geoscientists and Engineers Conference and Exhibition 2014 : Experience the Energy -Incorporating SPE EUROPEC 2014 (Amsterdam, Netherlands, 16-19 June 2014). C.357-361. 2014.

13. Thompson, M.; Arntsen, B.; Amundsen, L. Acquisition geometry versus 4C image quality: A study from Gullfaks South SEG Technical Program Expanded Abstracts. 2003, V. 22, iss. 1. P. 793-796

14. Martin, G.S.; Wiley, R.; Marfurt, K.J. Marmousi2: An elastic upgrade for Marmousi The Leading Edge. 2006, V. 25. P. 156-166. 